\title{
Selection of Local Thresholds for Tomogram Segmentation by Projection Distance Minimization
}

\author{
K. J. Batenburg and J. Sijbers \\ Vision Lab, Department of Physics \\ University of Antwerp, Belgium \\ joost.batenburg@ua.ac.be, jan.sijbers@ua.ac.be
}

\begin{abstract}
Segmentation is an important step to obtain quantitative information from tomographic data sets. To this end, global thresholding is often used in practice. However, it is usually not possible to obtain an accurate segmentation based on a single, global threshold. Instead, local thresholding schemes can be applied that use a varying threshold, depending on local characteristics of the tomogram. Selecting the best local thresholds is not a straightforward task, as local image features often do not provide sufficient information for choosing a proper threshold. Recently, the concept of projection distance was proposed as a new criterion for evaluating the quality of a tomogram segmentation. In this paper, we describe how Projection Distance Minimization (PDM) can be used to select local thresholds, based on the available projection data from which the tomogram was initially computed. By reprojecting the segmented image, a comparison can be made with the measured projection data. This yields a quantitative measure of the quality of the segmentation. By minimizing the difference between the computed and measured projections, optimal local thresholds can be computed.

Simulation experiments have been performed, comparing our local thresholding approach with an alternative local thresholding method and with optimal global thresholding. Our results demonstrate that the local thresholding approach yields segmentations that are significantly more accurate, in particular when the tomogram contains artifacts.
\end{abstract}

\section{Introduction}

Tomographic reconstructions, which are generally gray-scale images, are often segmented as to extract quantitative information, such as the shape or volume of image objects. Such segmentation is often performed by local or global thresholding [1]. However, the process of threshold selection is somewhat arbitrary and requires human interaction. Many algorithms have been proposed for selecting "optimal" thresholds with respect to various optimality measures [2. Global thresholds are typically selected from the histogram of the image, such that the distance between the image and the segmented image is minimized. Rosenfeld and Torre employ a method that is based on analyzing the concavity points on 
the convex hull of the image histogram. The deepest concavity points of the convex hull are potential candidates for the threshold [3]. Ridler and Calvard proposed an iterative, global thresholding method that models the gray-level distribution in an image as a mixture of two Gaussian distributions representing the background and foreground regions, respectively. Using the average of the foreground and background class means, a new threshold is established, which is iterated until convergence [4]. The clustering thresholding method of Otsu minimizes the weighted sum of intraclass variances of the foreground and background pixels to establish an optimum threshold [5]. Kapur et al. based their thresholding method on an entropy criterium. Thereby, the foreground and background classes are treated as two different sources [6]. The image is considered to be optimally thresholded when the sum of the two class entropies reaches a maximum.

Recently, Batenburg and Sijbers proposed a new approach for global threshold selection that makes use of the available tomographic projection data [7/8]. By reprojecting the segmented volume, the norm of the difference between the projections of the current segmentation and the measured projection data, called the projection distance, can be computed. This yields a quantitative measure of the quality of the segmentation. By minimizing the difference between the computed and measured projections (Projection Distance Minimization, or PDM), an optimal threshold can be computed. It was demonstrated in [8], that PDM leads to a significant improvement in segmentation accuracy, compared to histogram-based methods.

However, the capabilities of global threshold selection methods are limited by the maximum accuracy that can be obtained using global thresholding. If the tomogram exhibits variations in the intensity of certain image features, global thresholding can never lead to an accurate segmentation. For example, thick structures typically tend to be brighter than very thin structures in a tomogram, even if both structures consist of the same material in the original object. To account for local image variations, local thresholding methods were proposed. Abutaleb developed a local thresholding method based on the joint (2D) entropy of a pixel neighborhood 910. White and Rohrer developed a nonlinear, local thresholding method in which the gray value of the pixel is compared with the average of the gray values in a small neighborhood [11. If the pixel is significantly darker than the average, it is assigned as foreground; otherwise it is classified as background. Eikvil et al. developed a thresholding method in which a large window, with a small window positioned at its center, is moved across the image, and each pixel inside the small window is labeled on the basis of the clustering of the pixels inside the large window [12]. Blayvas et al. proposed an adaptive binarization method where the threshold is determined by interpolation of the image gray levels at points where the image gradient is high [13. The local thresholding method of Niblack adapts the local threshold according to the local mean and standard deviation over a sliding window [14.

Most of these adaptive thresholding methods that use a varying threshold for different regions of the image, lead to better results than global thresholding 
in some cases. However, selection of the local thresholds becomes increasingly difficult as the size of the regions is made smaller, as the local histogram is based on only a small number of pixels. Moreover, no objective criterion for the segmentation quality is available if only the information from the reconstructed image is used for segmentation.

In this paper, we propose an extension of the projection-based threshold selection method from [8, that uses a locally varying threshold field, instead of a single global threshold. The same optimization criterion, PDM, is now used to find an "optimal" threshold field. The threshold field is represented on a square grid that is coarser than the pixel grid of the tomogram. The thresholds for pixels that do not coincide with grid points in the coarse grid are computed by bilinear interpolation. Computing the threshold field for which the projection distance is minimal appears to be computationally hard. We describe how a minimum of the projection distance can be computed efficiently for the case that the threshold is only allowed to vary for a single grid point in the coarse grid, while keeping the threshold values fixed for the remaining grid points. By iterating this procedure several times for all coarse grid points, a local minimum of the projection distance is reached.

Simulation experiments have been performed, comparing the result of local thresholding based on PDM with the local thresholding method of Niblack [14] and with global thresholding based on PDM 8]. Our results demonstrate that the local thresholding approach yields segmentations that are significantly more accurate, in particular when the tomogram contains artifacts due to truncation of the projection data.

\section{Method}

For simplicity reasons, we restrict ourselves to two-dimensional tomograms. All concepts can be generalized to a 3D setting in a straightforward manner. Similar to algebraic reconstruction methods (i.e., ART, SART, see [15]) the tomogram is represented on a rectangular grid of width $w$ and height $h$. Put $n=w h$. The grey value image $\boldsymbol{v} \in \mathbb{R}^{n}$ that we want to segment is a tomographic reconstruction of some physical object, of which projections were acquired using a tomographic scanner. Projections are measured as sets of detector values for various angles, rotating around the object. Let $m$ denote the total number of measured detector values (for all angles) and let $\boldsymbol{p} \in \mathbb{R}^{m}$ denote the measured data. The physical projection process in tomography can be modeled as a linear operator $\boldsymbol{W}$ that maps the image $\boldsymbol{v}$ (representing the object) to the vector $\boldsymbol{p}$ of measured data:

$$
W v=p
$$

For parallel projection data, the operator $\boldsymbol{W}$ is a discretized version of the wellknown Radon transform. We represent $\boldsymbol{W}$ by an $m \times n$ matrix. From this point on, we assume that an image $\boldsymbol{v}$ has been computed that approximately satisfies Eq. (11). This image now has to be segmented using a locally varying threshold. 
In this paper, we focus on the segmentation of objects that consist of a single material, so that there are only two segmentation classes, for the object and the background. We assume that the material is homogenous, i.e., a perfect reconstruction of the original object should contain only two grey levels. However, most common tomographic reconstruction algorithms yield an image that consists of a range of grey levels, instead of a binary image, even if the object in the scanner is perfectly homogeneous. This becomes particularly noticeable if a reconstruction is computed from relatively few projections or if certain parts of the projection data are missing (e.g., truncated projections, where the object is larger than the field of view of the scanner). In such cases, the reconstruction problem is severely underdetermined, and many grey level images can have the same projections. Typically, continuous reconstruction algorithms do not use the prior knowledge about the discrete grey levels, but rather compute an image that contains many grey levels.

Even if prior knowledge about the two grey levels is not used in the reconstruction algorithm, this knowledge can still be exploited by the segmentation algorithm used after reconstruction. Our segmentation approach assigns a single real-valued grey value to both segmentation classes. The projections of the segmented image are then computed and compared to the measured projection data. The difference between the computed and measured projections provides a measure for the quality of the segmentation.

Although we assume that the original object consists of a single material, we do not assume prior knowledge of the actual grey levels of the background and the interior. These grey levels are treated as variables in the segmentation problem. We denote the grey level for the background and the interior of the object by $\rho_{1}$ and $\rho_{2}$, respectively. Put $\boldsymbol{\rho}=\left(\rho_{1} \rho_{2}\right)^{T}$.

We first define a segmentation problem where the local threshold can vary independently for each of the image pixels. The set of local thresholds for all pixels is represented by a vector $\tau \in \mathbb{R}^{n}$. We will refer to this vector as the threshold field.

For any $\boldsymbol{\rho} \in \mathbb{R}^{2}, t \in \mathbb{R}$, define the threshold function $r_{\boldsymbol{\rho}, t}: \mathbb{R} \rightarrow\left\{\rho_{1}, \rho_{2}\right\}$ by

$$
r_{\boldsymbol{\rho}, t}(v)=\left\{\begin{array}{ll}
\rho_{1} & (v \leq t) \\
\rho_{2} & (v>t)
\end{array} .\right.
$$

We also define the threshold function $r_{\boldsymbol{\rho}, \boldsymbol{\tau}}$ of an entire image $\boldsymbol{v} \in \mathbb{R}^{n}$, which yields a vector containing the thresholded pixel values:

$$
r_{\boldsymbol{\rho}, \boldsymbol{\tau}}(\boldsymbol{v})=\left(\begin{array}{lll}
\left.r_{\boldsymbol{\rho}, \tau_{1}}\left(v_{1}\right) \quad \ldots \quad r_{\boldsymbol{\rho}, \tau_{n}}\left(v_{n}\right)\right)^{T} .
\end{array}\right.
$$

For grey levels $\rho \in \mathbb{R}^{2}$ and a threshold field $\boldsymbol{\tau} \in \mathbb{R}^{n}$, define the projection difference $d(\boldsymbol{\rho}, \boldsymbol{\tau})$ by

$$
d(\boldsymbol{\rho}, \boldsymbol{\tau})=\left\|\boldsymbol{W} r_{\boldsymbol{\rho}, \boldsymbol{\tau}}(\boldsymbol{v})-\boldsymbol{p}\right\|_{2}
$$

The projection difference is used as the optimization criterion for finding the optimal threshold parameters. From this point, we will refer to this concept as Projection Difference Minimization (PDM). 
Problem 1. Let $\boldsymbol{W} \in \mathbb{R}^{m \times n}$ be a given projection matrix, let $\boldsymbol{v} \in \mathbb{R}^{n}$ be a grey level image and let $\boldsymbol{p} \in \mathbb{R}^{m}$ be a vector of measured projection data. Find $\boldsymbol{\tau} \in \mathbb{R}^{n}$ and $\boldsymbol{\rho} \in \mathbb{R}^{2}$, such that $d(\boldsymbol{\rho}, \boldsymbol{\tau})$ is minimal.

In Problem 1 the threshold for each pixel is allowed to vary independently. This means that the resulting segmentation class for each pixel $i$ (either background or interior) is independent of the grey value $v_{i}$, as the threshold $\tau_{i}$ can always be chosen either smaller or larger than $v_{i}$. In fact, this threshold selection problem is equivalent to a reconstruction problem from Discrete Tomography, where the main objective is to reconstruct a binary image from its projections [16. Although solving this discrete tomography problem can lead to very accurate segmentation results, even if few projection are used, the problem is computationally very hard (see, e.g., [17]). In cases where it is relatively easy to acquire more projection images, continuous tomography followed by thresholding (either local or global) is often preferable.

At the other end of the granularity spectrum is the case where all entries of $\boldsymbol{\tau}$ must have the same value, i.e., global thresholding. This approach was already proposed in 8. For binary images, it was demonstrated that only the global threshold $\tau$ has to be optimized, as the optimal grey values $\rho_{1}$ and $\rho_{2}$ can be computed directly once the threshold $\tau$ has been set.

In this paper, we focus on a segmentation problem that can be considered as an "intermediate" problem, between discrete tomography and global thresholding based on PDM. Instead of allowing the threshold field $\boldsymbol{\tau}$ to vary independently for each pixel, the value of the threshold is specified on a coarse grid, which is superimposed on the pixel grid of the image $\boldsymbol{v}$. The threshold value for each pixel of $v_{i}$ is then computed by bilinear interpolation from the set threshold values. In this way, the local thresholds will vary only gradually, while the threshold field can still vary significantly throughout the image. The choice for bilinear interpolation is mainly motivated by computational convenience. More sophisticated interpolation schemes (i.e., bicubic interpolation) may lead to better results. However, such schemes typically yield more variables in the resulting optimization problem.

Figure 1 illustrates how the coarse grid is superimposed onto the finer pixel grid of the image $\boldsymbol{v}$. Note that only a small portion of the image is depicted. As an example, suppose that the thresholds are given for the four points indicated in the figure (with corresponding threshold values $t_{1}, \ldots, t_{4}$. Let the $(x, y)$-coordinates of these four points be given by $(0,0),(1,0),(1,1)$ and $(0,1)$, respectively. We refer to the four squares between $(0,0)$ and its surrounding coarse grid points as the quadrants surrounding $(0,0)$. For any pixel $p$ with center $\left(x_{p}, y_{p}\right)$ in the topright quadrant, the threshold $t_{p}$ is defined by

$$
t_{p}=\left(1-x_{p}\right)\left(1-y_{p}\right) t_{1}+x_{p}\left(1-y_{p}\right) t_{2}+\left(1-x_{p}\right) y_{p} t_{3}+x_{p} y_{p} t_{4} .
$$

Let $k$ be the total number of grid points on the coarse interpolation grid. We refer to the vector of thresholds for these points by $\tau^{\prime} \in \mathbb{R}^{k}$. The mapping $I: \mathbb{R}^{k} \rightarrow \mathbb{R}^{n}$ assigns the corresponding interpolated threshold to each pixel in the fine grid: 


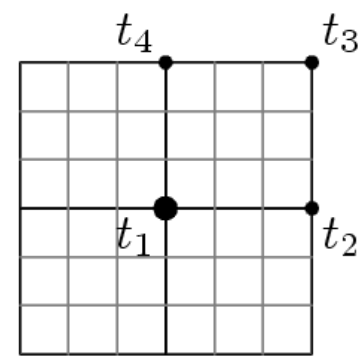

Fig. 1. A coarse grid is superimposed on the finer pixel grid of the reconstructed image. The thresholds are only specified in the coarse grid points. The threshold for each individual pixel is computed by bilinear interpolation.

$$
\boldsymbol{\tau}=I\left(\boldsymbol{\tau}^{\prime}\right)
$$

Using these definitions, we can now formulate the central problem of this paper:

Problem 2. Let $\boldsymbol{W} \in \mathbb{R}^{m \times n}$ be a given projection matrix, let $\boldsymbol{v} \in \mathbb{R}^{n}$ be a grey scale image and let $\boldsymbol{p} \in \mathbb{R}^{m}$ be a vector of measured projection data. Find $\boldsymbol{\tau}^{\prime} \in \mathbb{R}^{k}$ and $\boldsymbol{\rho} \in \mathbb{R}^{2}$, such that $d\left(\boldsymbol{\rho}, I\left(\boldsymbol{\tau}^{\prime}\right)\right)$ is minimal.

We will now describe how a constrained version of Problem 2 can be solved efficiently, where only one of the entries of $\boldsymbol{\tau}^{\prime}$ is allowed to vary, while the remaining entries are kept fixed. Again, consider the example from Figure 1. Suppose that all thresholds on the coarse grid are kept fixed, except for $t_{1}$. The only pixels for which the thresholds are affected by a change of $t_{1}$ are those in the four quadrants surrounding $t_{1}$, as shown in the figure.

For a pixel $p$ with grey level $v_{p}$ and center $\left(x_{p}, y_{p}\right)$ in the topright quadrant, we have

$$
\begin{aligned}
& v_{p} \geq t_{p} \Longleftrightarrow \\
& v_{p} \geq\left(1-x_{p}\right)\left(1-y_{p}\right) t_{1}+x_{p}\left(1-y_{p}\right) t_{2}+\left(1-x_{p}\right) y_{p} t_{3}+x_{p} y_{p} t_{4} \Longleftrightarrow \\
& \frac{v_{p}-x_{p}\left(1-y_{p}\right) t_{2}-\left(1-x_{p}\right) y_{p} t_{3}-x_{p} y_{p} t_{4}}{\left(1-x_{p}\right)\left(1-y_{p}\right)} \geq t_{1} .
\end{aligned}
$$

The term $g(p)=\frac{v_{p}-x_{p}\left(1-y_{p}\right) t_{2}-\left(1-x_{p}\right) y_{p} t_{3}-x_{p} y_{p} t_{4}}{\left(1-x_{p}\right)\left(1-y_{p}\right)}$ is called the relative grey level of $p$ with respect to $t_{1}$. The equation defining the relative grey level of pixels w.r.t. $t_{1}$ is different for each of the quadrants surrounding the coarse grid point. Eq. (17) shows that the problem of finding a solution of Problem 2 in case only one entry of $\boldsymbol{\tau}^{\prime}$ is allowed to vary, can be considered as a variant of the global thresholding problem from 8]. In this global thresholding problem, only pixels in the four quadrants surrounding the variable entry of $\boldsymbol{\tau}^{\prime}$ have to be considered (as the remaining pixels are unaffected by a change of this threshold) and the relative grey level of each surrounding pixel is used instead of the grey levels from $v$. 
For any fixed $\boldsymbol{\tau}$, every entry of the vector $\boldsymbol{W} r_{\boldsymbol{\rho}, \boldsymbol{\tau}}(\boldsymbol{v})$ reduces to a linear expression in $\rho_{1}$ and $\rho_{2}$. Consequently, for fixed $\boldsymbol{\tau}$, the projection difference $d(\boldsymbol{\rho}, \boldsymbol{\tau})$ reduces to a second-degree polynomial in $\rho_{1}$ and $\rho_{2}$. In fact, this property holds for any segmentation of $\boldsymbol{v}$ (not just those obtained by thresholding). In [8], it is shown that for any fixed segmentation of the image $\boldsymbol{v}$, the projection difference $d(\boldsymbol{\rho})$ can be written in the form

$$
d(\boldsymbol{\rho})^{2}=|\boldsymbol{p}|^{2}+\overline{\boldsymbol{c}}^{T} \boldsymbol{\rho}+\boldsymbol{\rho}^{T} \overline{\boldsymbol{Q}} \boldsymbol{\rho} .
$$

(i.e., a second-degree polynomial in $\rho_{1}$ and $\rho_{2}$ ), where $\overline{\boldsymbol{Q}} \in \mathbb{R}^{\ell \times \ell}$ matrix and $\overline{\boldsymbol{c}} \in \mathbb{R}^{2}$. Explicit expressions for the matrix $\overline{\boldsymbol{Q}}$ and vector $\overline{\boldsymbol{c}}$ are also described. Once $\overline{\boldsymbol{Q}}$ and $\overline{\boldsymbol{c}}$ are known, the vector $\boldsymbol{\rho}$ for which the projection distance is minimal can be computed by minimizing the quadratic polyomial in Eq. (8). It is also shown in [8] how $\overline{\boldsymbol{Q}}$ and $\overline{\boldsymbol{c}}$ can be updated efficiently if the segmentation class for a single pixel is changed. This update step is independent of the algorithm that is used to compute the segmentation, so it can be used in our new local threshold method without much modification. Figure 2 shows the basic steps for solving the variant of Problem 2 where only one of the entries of $\boldsymbol{\tau}^{\prime}$ is allowed to vary.

Computing a global minimum of $d\left(\boldsymbol{\rho}, I\left(\boldsymbol{\tau}^{\prime}\right)\right)$ as stated in Problem 2 appears to be computationally very hard. In fact, if the coarse grid is taken to have the same resolution as the pixel grid of the reconstructed image, solving Problem 2 is equivalent to solving a variant of the discrete tomography problem. For certain weight matrices $\boldsymbol{W}$, this problem is known to be NP-hard [17].

Instead, we propose an iterative algorithm that is guaranteed to converge to a local minimum of the projection distance. The basic steps of this algorithm are shown in Figure 3 . In each iteration, a random grid point on the coarse grid is selected. The optimal threshold for this grid point is computed, while keeping the thresholds in all other coarse grid points fixed. The algorithm terminates if no improvement has been found after $K$ iteration, where $K$ is a constant integer. Although this algorithm may not find a global minimum of the projection distance, the projection distance of the resulting segmentation can never be worse than the projection distance found using global thresholding. The best global threshold is used to initialize the local thresholds in the coarse grid points and in each iteration the projection distance does not increase.

\section{Results}

Simulation experiments have been performed, starting from two phantom images: a vascular structure (referred to as 'vessel image') and a femur image, shown in Fig. 4(a) and Fig 4(b), respectively. For each experiment, simulated parallel beam projections have been computed using equally spaced projection angles. Based on the projection data, a reconstruction was computed using the SART algorithm (cfr. [15). The resulting SART reconstructions are shown in Fig. 5(a)t 5(c) Fig. 5(a) and Fig. 5(b) were generated from 180 projections, while Fig 5(c) 


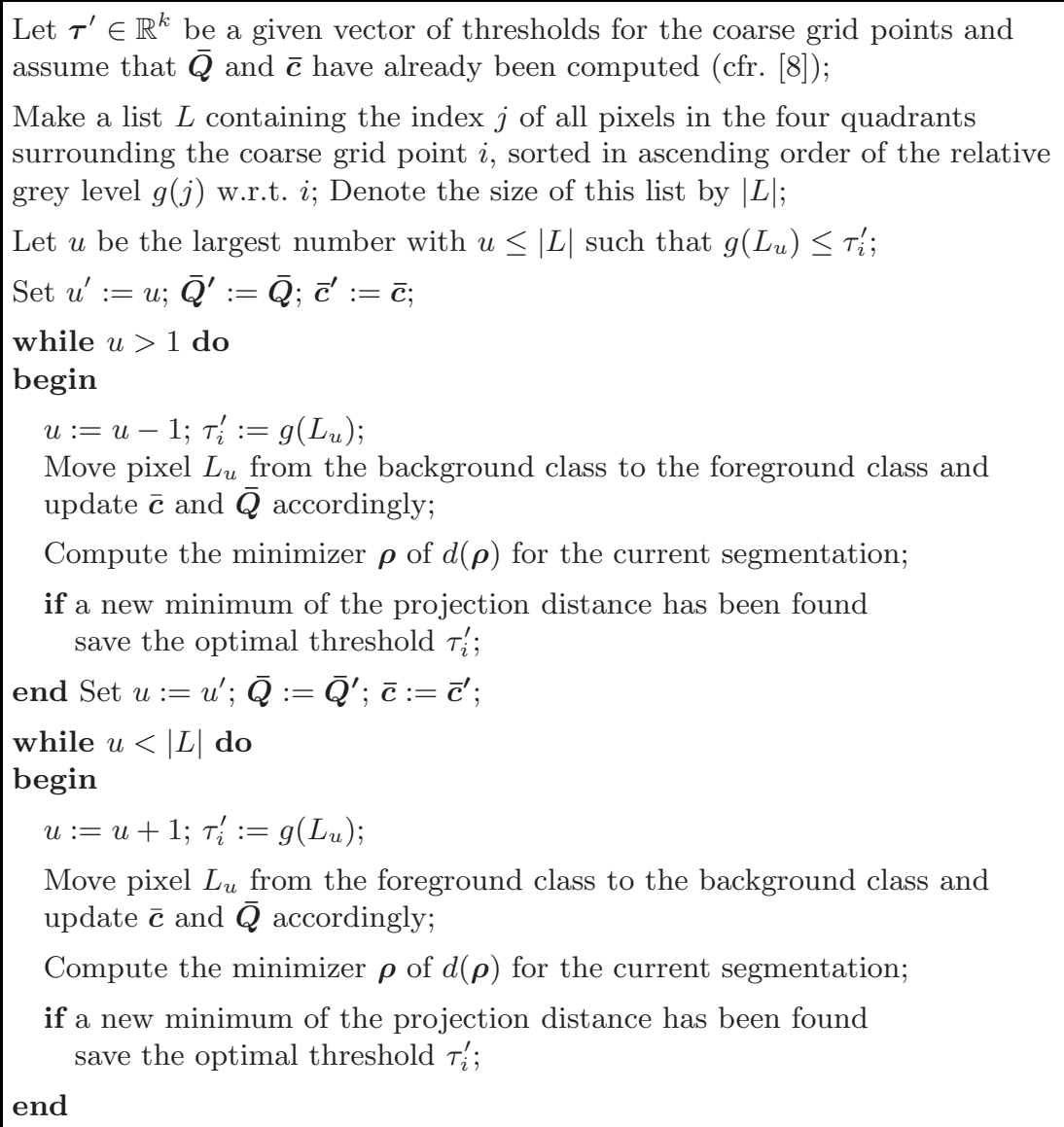

Fig. 2. Basic steps for finding the optimal threshold in a given coarse grid point $i$, while keeping the thresholds in all remaining coarse grid points fixed

shows the SART reconstruction from 180 truncated projections (i.e., the simulated beam is more narrow than the object). The truncated projections cause artifacts in the reconstruction, which are particularly difficult to segment using a single global threshold, as they result in significant grey value variations. For all local threshold experiments in this section, a spacing of 16 pixels between consecutive coarse grid points was used.

In a first phase, the results of our proposed local thresholding approach are compared with global thresholding. For global thresholding, we used the PDM algorithm from [8], which computes a global minimum of the projection distance and which was proved to yield significantly better results than conventional global thresholding techniques. The global PDM thresholding results are shown in Fig. $5(\mathrm{~g})+5(\mathrm{i})$. 
compute the optimal global threshold $\tau$, using the algorithm described in [8];

Set $\tau_{i}^{\prime}=\tau$ for each point $i=1, \ldots, k$ on the coarse grid;

repeat

select a random threshold $\tau_{i}^{\prime}$ on the coarse grid;

compute the optimal projection difference $d\left(\boldsymbol{\rho}, I\left(\boldsymbol{\tau}^{\prime}\right)\right)$ that can be obtained by changing $\tau_{i}^{\prime}$, while keeping the values of the other thresholds fixed;

set the threshold $\tau_{i}^{\prime}$ to its new optimal value (changing the local threshold field); until (no improvement was made during the last $K$ iterations;

Fig. 3. Basic steps of the threshold selection algorithm. The variable $K$ in the outer loop condition refers to a constant integer.

Table 1. Comparison of the local thresholding performance of the Niblack and PDM method. The numbers represent the number of different pixels between the thresholded image and the original phantom image.

\begin{tabular}{|c|c|c|c|}
\hline & Vessel & Femur & Femur (trunc) \\
\hline Niblack & 9977 & 3142 & 10694 \\
PDM (global) & 3374 & 1365 & 10743 \\
PDM (local) & 2967 & 1182 & 7584 \\
\hline
\end{tabular}

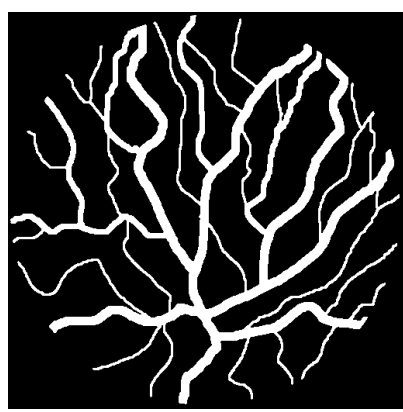

(a) Vessel image

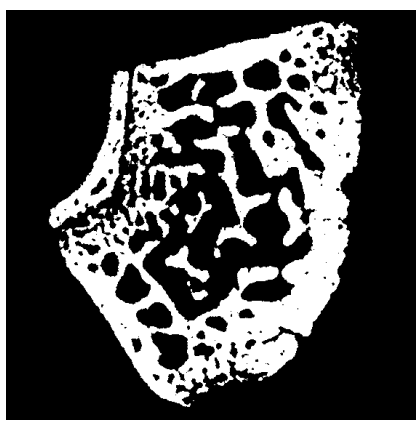

(b) Femur image

Fig. 4. Phantom images used in our simulation experiments: (a) vessel image and (b) femur image

Next, the SART reconstructions were segmented using the local thresholding method of Niblack 14, which is commonly used as a reference for performance evaluation. The Niblack method adapts the local threshold according to the local mean and standard deviation of a sliding window. The method depends on two parameters: the width of the sliding window and the threshold weight of the 


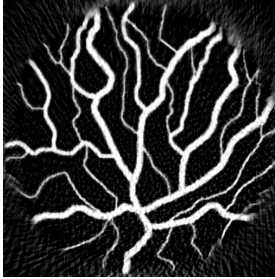

(a)

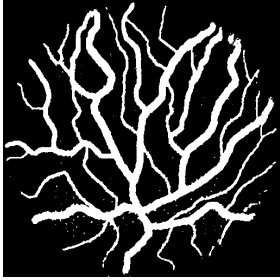

(d)

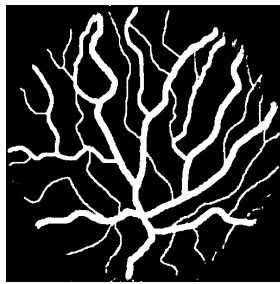

(g)

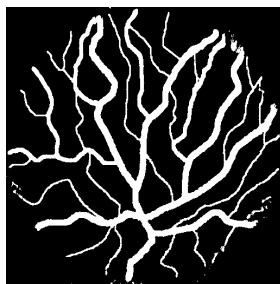

(j)

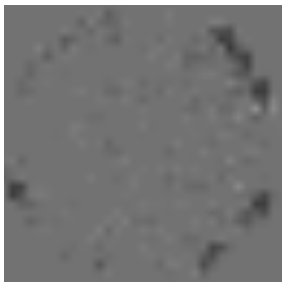

$(\mathrm{m})$

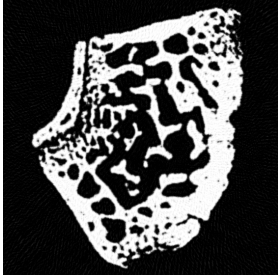

(b)

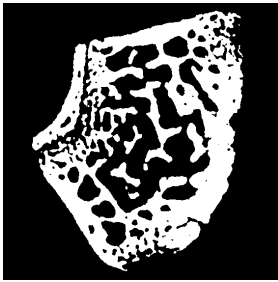

(e)

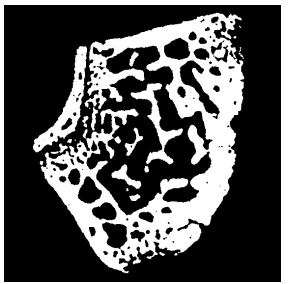

(h)

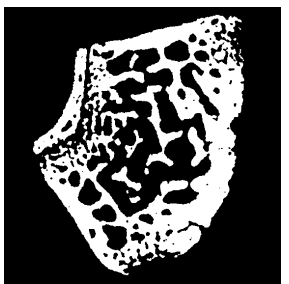

$(\mathrm{k})$

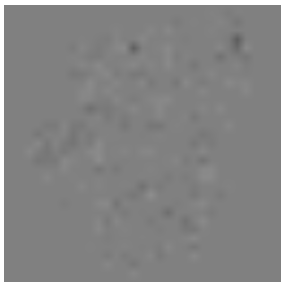

$(n)$

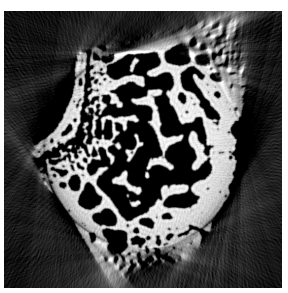

(c)

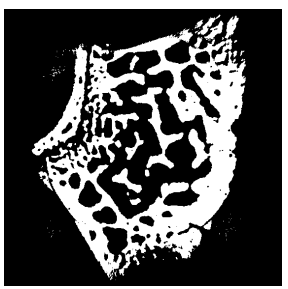

(f)

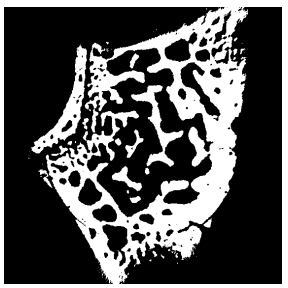

(i)

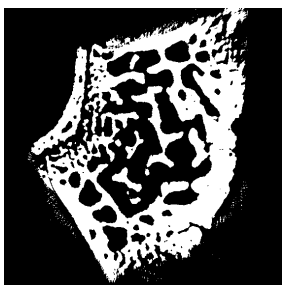

(1)

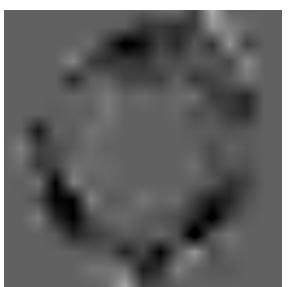

(o)

Fig. 5. (a-c) SART reconstructions from (a-b) 180 projections and (c) 180 truncated projections; (d-f) Niblack local thresholding results; (g-i) global PDM thresholding result; (j-l) local PDM thresholding result; (m-o) PDM threshold fields 
standard deviation. In practice, these Niblack parameters cannot be optimized because of the lack of ground truth. In our simulation experiments, in which the ground truth was available, we selected the window width and the weight parameter such that the difference between the thresholded result and the original was minimal.

Finally, the SART reconstructions shown in Fig. 5(a) 5(c) were segmented using the local PDM thresholding scheme as proposed in this paper. In all three cases, our local thresholding algorithm results in a significant reduction of the number of pixel errors. The running time of each test was around $30 \mathrm{~s}$, on a Pentium IV PC running at $3 \mathrm{GHz}$. The thresholding results of the local PDM method are shown in Fig. 5(j) $5(\mathrm{l})$ respectively. The corresponding threshold fields generated from the local PDM method are shown in Fig $5(\mathrm{~m}) 5(\mathrm{o})$ respectively. These fields were formed by bilinear interpolation from the threshold values $\tau^{\prime}$ on the coarse grid.

To quantify the performance of each segmentation method, for each method the total number of pixel errors was determined, which was computed from the original phantom. The quantitative thresholding results are shown in Table 1 . From this table, it is clear that the proposed local PDM thresholding method outperforms the other methods with respect to the pixel error.

\section{Conclusion}

Local grey value thresholding is a common segmentation procedure. However, finding the optimal grey level thresholds is far from trivial. Many procedures have been proposed to select the thresholds based on various image features. Unfortunately, these methods suffer from a clear objective threshold selection criterion.

In our paper, we have presented an innovative approach, called local PDM (Projection Distance Minimization), to find the optimal threshold grey levels by exploiting the available projection data. Reprojection of the segmented image and subsequent comparison with the measured projection data, yields an objective criterion for the quality of a segmentation. Our approach aims at minimizing the projection distance.

The experimental results show that the proposed local PDM method results in a small difference between the original object and the reconstruction. Simulation experiments were performed for three SART reconstructions of phantom images. In all test cases, PDM clearly leads to significantly better segmentation results than both the Niblack method for local segmentation and global thresholding based on PDM.

\section{Acknowledgement}

This work was financially supported by the F.W.O. (Fund for Scientific Research

- Flanders, Belgium) 


\section{References}

1. Eichler, M.J., Kim, C.H., Müller, R., Guo, X.E.: Impact of thresholding techniques on micro-CT image based computational models of trabecular bone. In: ASME Advances in Bioengineering, September 2000, vol. 48, pp. 215-216 (2000)

2. Glasbey, C.A.: An analysis of histogram-based thresholding algorithms. Graphical Models and Image Processing 55(6), 532-537 (1993)

3. Rosenfeld, A., Torre, P.: Histogram concavity analysis as an aid in threshold selection. IEEE Trans. Syst., Man, Cybern. 13, 231-235 (1983)

4. Ridler, T.W., Calvard, S.: Picture thresholding using an iterative selection method. IEEE Transactions on Systems, Man, and Cybernetics 8, 630-632 (1978)

5. Otsu, N.: A threshold selection method from gray level histograms. IEEE Trans. Syst., Man, Cybern. 9, 62-66 (1979)

6. Kapur, J., Sahoo, P., Wong, A.: A new method for gray-level picture thresholding using the entropy of the histogram. Comp Vision, Graph, and Image Proc. 29(3), 273-285 (1985)

7. Batenburg, K.J., Sijbers, J.: Automatic multiple threshold scheme for segmentation of tomograms. In: Pluim, Josien, P.W., Reinhardt, J.M. (eds.) Proceedings of SPIE Medical Imaging, San Diego, CA, USA, February 2007, vol. 6512 (2007)

8. Batenburg, K.J., Sijbers, J.: Automatic threshold selection for tomogram segmentation by reprojection of the reconstructed image. In: Kropatsch, W.G., Kampel, M., Hanbury, A. (eds.) CAIP 2007. LNCS, vol. 4673, pp. 563-570. Springer, Heidelberg (2007)

9. Abutaleb, A.S.: Automatic thresholding of grey-level pictures using twodimensional entropies. Pattern Recognition 47, 22-32 (1989)

10. Brink, A.D.: Thresholding of digital images using two-dimensional entropies. Pattern Recognition 25, 803-808 (1992)

11. White, J.M., Rohrer, G.D.: Image thresholding for optical character recognition and other applications requiring character image extraction. IBM Journal of Research and Development 27(4), 400-411 (1983)

12. Eikvil, L., Taxt, T., Moen, K.: A fast adaptive method for binarization of document images. In: Proceedings of the International Conference on Document Analysis and Recognition, pp. 435-443 (1991)

13. Blayvas, I., Bruckstein, A., Kimmel, R.: Efficient computation of adaptive threshold surfaces for image binarization. Pattern Recognition 39(1), 89-101 (2006)

14. Niblack, W.: An introduction to image processing. Englewood Cliffs, New York (1986)

15. Kak, A.C., Slaney, M.: Principles of Computerized Tomographic Imaging. Volume Algorithms for reconstruction with non-diffracting sources, pp. 49-112. IEEE Press, New York (1988)

16. Herman, G.T., Kuba, A. (eds.): Advances in Discrete Tomography and Its Applications. Applied and Numerical Harmonic Analysis. Birkhäuser, Boston (2007)

17. Gardner, R., Gritzmann, P., Prangenberg, D.: On the computational complexity of reconstructing lattice sets from their X-rays. Discrete Math. 202, 45-71 (1999) 